\title{
Association between Self-Reported Sleep Duration and Dietary Nutrients in Korean Adolescents: A Population-Based Study
}

\author{
Jee Hyun Lee ${ }^{1}\left(\mathbb{D}\right.$, Sang-Jin Chung ${ }^{2}(\mathbb{D})$ and Won Hee Seo ${ }^{1, *(\mathbb{C})}$ \\ 1 Department of Pediatrics, Korea University Ansan Hospital, Korea University College of Medicine, \\ Ansan 15459, Korea; izzihn@gmail.com \\ 2 Department of Foods and Nutrition, Kookmin University, Seoul 02707, Korea; schung@kookmin.ac.kr \\ * Correspondence: wonny508@korea.ac.kr; Tel.: +82-31-412-5096
}

Received: 13 September 2020; Accepted: 6 November 2020; Published: 8 November 2020

\begin{abstract}
Background: Adolescence is a transient period from childhood to adulthood, which is characterized by rapid physical growth and psychological changes, including sleep. Because the relationship between insufficient sleep and obesity has been observed in children and adults, the potential links between sleep, dietary intake, and nutrition have received increased attention. We aimed to examine the association of sleep duration with dietary nutrients intake in South Korean adolescents; (2) Methods: This population-based, cross-sectional study analyzed the data obtained from the Korea National Health and Nutrition Examination Survey between 2013 and 2015. Data related to 1422 adolescents aged 12-18 years (741 males and 681 females) were included in the analysis. Sleep duration was assessed using a self-reported questionnaire. Nutrient intake, including daily total energy intake, was assessed with a 24-h dietary recall questionnaire; (3) Results: Most males $(84.4 \%)$ and females $(86.4 \%)$ reported $<9$ h of sleep per night. Short sleep duration was inversely associated with body mass index and obesity in both sexes. We found that higher intake of fiber and lower intake of sodium were associated with longer sleep duration $(P<0.05)$. When comparing the intake above and below the estimated average requirements (EAR), the difference in sleep duration was significant in the group that consumed vitamins $B_{1}$ and $C$ below EAR; (4) Conclusions: The findings of this study indicate that sleep duration can be associated with intake of some nutrients, which may also be associated with obesity in adolescents. Therefore, it is possible to prevent obesity and its complications by controlling the sleep duration and intake of nutrients of adolescents.
\end{abstract}

Keywords: adolescent; nutrients; obesity; sleep duration

\section{Introduction}

Adolescence is a transient period from childhood to adulthood, which is characterized by rapid physical growth and psychological changes, including sleep. Sleep architecture changes throughout adolescence, showing a marked reduction in slow wave sleep (SWS) and rapid eye movement (REM) sleep in absolute terms, but not as a percentage of total sleep time [1]. Moreover, short sleep duration in adolescence is influenced by external factors such as use of electronic devices and social networking [2]. However, the sleep need does not change during adolescence-the estimated median sleep length necessary for adolescents to sustain waking vigilance and alertness is $9 \mathrm{~h}[3,4]$. Insufficient sleep may interfere with the physiological restoration that occurs during this period, leading to biological and behavioral risk factors for chronic disease development including obesity, depression, and cardiovascular disease [5-8]. 
The rates of obesity among adolescents have increased over the past 3 decades; currently, more than 330 million children and adolescents worldwide were overweight or obese [9]. Obesity during adolescence is a predictor of obesity in adulthood [10]. Therefore, prevention efforts should begin early in life to prevent complication of obesity in adulthood. Since the relationship between insufficient sleep and obesity has been observed in both children and adults, the potential links between sleep, dietary intake, and nutrition have received increased attention. Although the exact mechanism of association between dietary intake and sleep duration is not clear, several possibilities have been suggested as follows: (1) short sleep duration increased free time to eat foods [11]; (2) sleep deprivation has been associated with activation of the stress system, which has been associated with decreased leptin and elevated ghrelin levels and increased insulin insensitivity [12,13], and a short sleep duration has been associated with decreased levels of thyroid stimulating hormone and growth hormone that stimulate the basal metabolic rate [14,15], whereas some studies have reported that manipulating the diet can alter sleep duration and quality. High intake of fish and vegetables was associated with good sleep quality [16]. The Mediterranean diet was associated with insomnia in older adults [17].

There have been studies on the association between dietary habits or food intake, such as intake of sweetened food or beverages, and sleep duration, but there are few studies on the association between nutrients intake and sleep duration in adolescents. Therefore, this study examined the association between sleep duration and dietary nutrients intake presented in the results of the 6th Korean Nutrition Health and Nutrition Examination Survey (KNHANES).

\section{Materials and Methods}

\subsection{Survey Overview and Study Subjects}

This population-based, cross-sectional study analyzed the data of South Korean adolescents who participated in the Korea National Health and Nutrition Examination Survey (KNHANES) between 2013 and 2015. The KNHANES, a nationwide ongoing survey of non-institutionalized civilians, has been conducted by the Division of Chronic Disease Surveillance of the Korea Centers for Disease Control and Prevention (KCDC) and the Korean Ministry of Health and Welfare since 1998 [18]. The survey was designed to assess national health and nutritional status and consists of a health interview, nutritional assessment, and health examination. Participants were randomly selected from sampled household units using a stratified, multistage, and probability-based sampling design based on the component ratio of population and the housing census from the 2005 National Census Registry in South Korea.

Of 22,948 participants sampled in the KNHANES 2013-2015, we excluded 21,093 individuals who were aged $<12$ years or $>18$ years and 433 individuals who had missing values for major target variables, such as nutritional survey and sleep duration, and anthropometric measures. A total of 1422 adolescents (741 males and 681 females) were analyzed. All study subjects were considered minors; therefore, their parents signed the written informed consent forms. The institutional review board of the KCDC approved the study protocol. The survey data are available from the KCDC website (http://knhanes.cdc.go.kr).

\subsection{Sleep Duration Measurements}

Sleep duration was assessed using a self-reported questionnaire. All subjects were asked about their sleep duration, smoking status, alcohol consumption, physical activity, and monthly household income level. Sleep duration data was acquired from the following question in a self-reported questionnaire: "How many hours do you sleep on average?" Sleep duration was classified into five categories- $\leq 5 \mathrm{~h}, 6 \mathrm{~h}, 7 \mathrm{~h}, 8 \mathrm{~h}$, and $\geq 9 \mathrm{~h}$. 


\subsection{Lifestyle Variables and Nutritional Assessment}

Data on age, area of residence, education level, occupation, monthly household income level, physical activity, smoking status, and alcohol consumption were obtained during the health-related interview. We divided the subjects into non-smokers and current smokers according to their self-reported smoking status. Subjects who had smoked cigarettes at least once during the month before the survey were defined as current smokers. Regarding alcohol consumption, subjects who had at least one alcoholic drink once during the month before the survey were defined as alcohol drinkers. Physical activity was assessed using minutes of walking per day calculated from weekly frequency and minutes of walking. Monthly household income level was divided into the lower 25th percentile of the total subjects or higher.

\subsection{Anthropometry and Body Composition Measurements}

Trained staff members performed anthropometric and biochemical measurements. Height and body weight $(\mathrm{BWt})$ were measured to the nearest $0.1 \mathrm{~cm}$ and $0.1 \mathrm{~kg}$, respectively, while the participants were barefoot and wearing light clothing. Waist circumference was measured at the narrowest point between the lower border of the rib cage and the iliac crest while the participants were in standing position. Body mass index (BMI) was calculated by dividing BWt $(\mathrm{kg})$ by height $(\mathrm{m})$ squared.

BMI was categorized into 4 groups according to American Medical Association Expert Committee Recommendations [19]: underweight (BMI $<5$ th percentile), healthy weight (BMI between the 5th and 84th percentiles), overweight (BMI between the 85th and 94th percentiles), and obese (BMI $\geq 95$ th percentile).

\subsection{Nutrients Intake Asessment}

Nutrient intake, including daily total energy intake, was assessed using a 24-h dietary recall questionnaire administered by a trained dietician. The $24-h$ recall method examines the type and intake of food consumed one day before the survey date using tools such as 2D images, measuring cups, and thick slice. Energy and nutrient intakes for each participant were calculated using the Korean Foods and Nutrients Database of the Rural Development Administration [20]. Food groups were analyzed into 18 categories based on the Standard Food Composition Table, 8th Revision in Korea [21]. The amount of nutrients intake was compared with the Dietary Reference Intakes for Koreans 2015 [22].

\subsection{Statistical Analyses}

Statistical analyses were performed with the statistical software package (SAS version 9.4 for Windows, SAS Institute, Cary, NC, USA) using SAS survey procedures including the appropriate weight, strata, domain, and cluster variables to account for the complex multistage sampling design in the KNHANES. Continuous variables (presented as mean \pm standard error) were compared by sex using the independent t-test and by categories of sleep duration using the analysis of variance (ANOVA). To evaluate the appropriateness of nutrient intake, the intake of nutrients was divided into two groups (below the estimated average requirement (EAR) and above EAR) to test the association with sleep duration using the chi-square test [22].

Least square means of the sleep duration were compared according to BMI quartiles using the analysis of covariance (ANCOVA) after adjustment for age, smoking status, alcohol consumption, physical activity, monthly household income level, and energy intake (Figure 1).

ANCOVA was used with calculation of linear trend to evaluate the relationship between dietary nutrients and sleep duration while controlling for other potential confounders. Model 1 was unadjusted. In Model 2, adjustments were made for the variables age and sex. Model 3 was adjusted for the variables of Model 2, smoking status, alcohol consumption, physical activity, monthly household income level, and energy intake. $P$ values were two tailed, and statistical significance was set at $P<0.05$. 


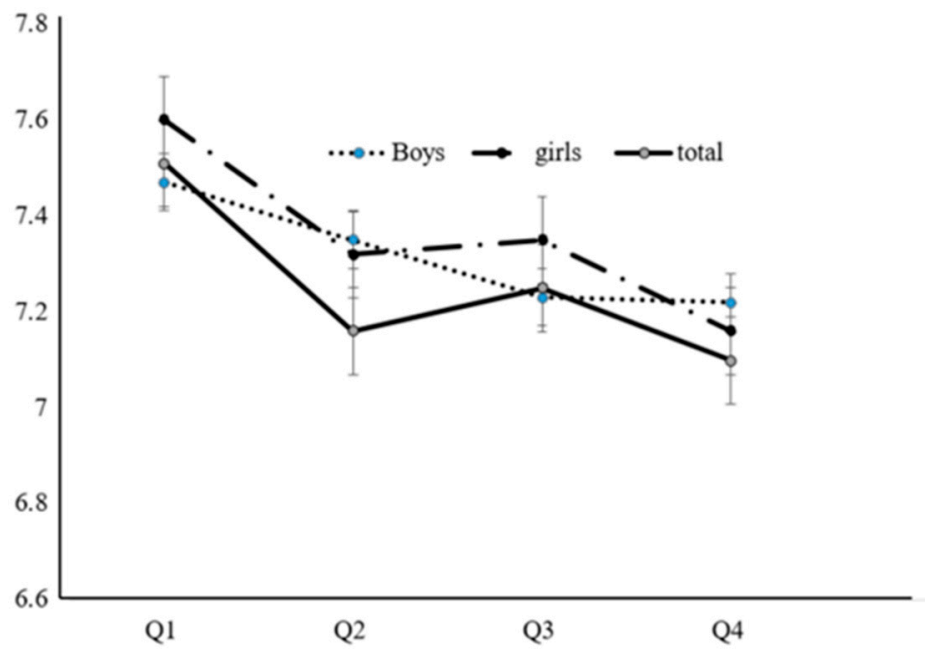

Figure 1. Adjusted sleep duration according to body mass index in both sexes. BMI: Body mass index. Adjusted for age, smoking, alcohol, physical activity, income, energy intake, each $P$ value $<0.05$. Q1: lowest BMI quartiles, Q2: middle-low BMI quartiles, Q3 middle-high BMI quartiles, Q4: highest BMI quartiles.

\section{Results}

\subsection{Demographic Characteristics of Study Participants}

Baseline characteristics of the study population are presented in Table 1 . The study participants consisted of 741 males and 681 females aged 12-18 (average, 15) years; 62.5\% were aged 12-15 years and $37.5 \%$ were aged 16-18 years. Regarding BMI, $19.0 \%$ of participants were above the 85 th percentile, and the average BMI was 21.7.

Table 1. Baseline characteristics of study subjects.

\begin{tabular}{cccc}
\hline Study Population $(\mathbf{n}=\mathbf{1 4 2 2})$ & Male $(\mathbf{n}=\mathbf{7 4 1})$ & Female $(\mathbf{n}=\mathbf{6 8 1})$ & $\boldsymbol{P}$ \\
\hline Age $($ years $)$ & $15.1 \pm 0.07$ & $15.1 \pm 0.08$ & 0.747 \\
Height $(\mathrm{cm})$ & $169.3 \pm 0.36$ & $159.8 \pm 0.27$ & $<0.001^{*}$ \\
Weight $(\mathrm{kg})$ & $62.6 \pm 0.58$ & $54.3 \pm 0.47$ & $<0.001^{*}$ \\
Waist Circumference $(\mathrm{cm})$ & $74.0 \pm 0.43$ & $69.3 \pm 0.36$ & $<0.001^{*}$ \\
BMI $\left(\mathrm{kg} / \mathrm{m}^{2}\right)$ & $21.7 \pm 0.18$ & $21.2 \pm 0.15$ & $0.020^{*}$ \\
Energy intake (cal) & $2567.2 \pm 50.7$ & $1890.5 \pm 33.4$ & $<0.001^{*}$ \\
Fat intake (\%) & $24.9 \pm 0.4$ & $24.0 \pm 0.4$ & 0.085 \\
Walking activity (per day) & $33.8 \pm 1.68$ & $31.0 \pm 1.49$ & 0.195 \\
Sleep Duration (hours, \%) & & & $<0.001^{*}$ \\
$<6 \mathrm{~h}$ & 9.7 & 16.7 & \\
6 to $<8 \mathrm{~h}$ & 47.5 & 50.3 & \\
8 to $<9 \mathrm{~h}$ & 27.2 & 19.4 & \\
$\geq 9 \mathrm{~h}$ & 15.6 & 13.6 & \\
\hline
\end{tabular}

Values are presented as the mean \pm standard error or percentage, ${ }^{*} P<0.05$.

Only $14.7 \%(n=215)$ of participants reported more than 9 h sleep per night, with less than half of them (males, $42.8 \%$; females, $33.0 \%$ ) reporting below $8 \mathrm{~h}$ of sleep per night. Sleep duration differed significantly between sexes $(P<0.001)$.

\subsection{Sleep Duration and Obese Status}

Figure 1 shows the sleep duration according to the quartiles of BMI. Sleep duration was inversely associated with BMI and obesity in both sexes $(P<0.05)$. 


\subsection{Sleep Duration and Dietary Nutrients Assessments}

There were no associations between sleep duration and total energy intake and intake of fat, carbohydrate, and protein (Tables 2 and 3). After adjustment for confounding factors (age, smoking status, alcohol consumption, physical activity, monthly household income level, and energy intake), fiber and sodium intake were significantly associated with sleep duration. Higher intake of fiber and lower intake of sodium were associated with longer sleep time $(P<0.05$, Table 3$)$. When comparing the intake above and below EAR, the difference in sleep duration was significant in the group that consumed vitamins B1 and C below EAR (Table 3).

Model 1 was unadjusted. Model 2 was adjusted for age. Model 3 was adjusted for age, smoking, alcohol, physical activity, income and energy intake.

Table 2. Comparison between amount of dietary nutrient intake and sleep duration.

\begin{tabular}{|c|c|c|c|c|c|c|c|c|}
\hline Sleep Duration & & & & $<6 \mathrm{~h}$ & 6 to $<8 \mathrm{~h}$ & 8 to $<9 \mathrm{~h}$ & $\geq 9 \mathrm{~h}$ & $P$ \\
\hline N (\%) & Intake & $\mathbf{n}$ & $\%$ & $157(13.1)$ & $690(48.8)$ & $360(23.4)$ & $215(14.7)$ & \\
\hline \multirow{2}{*}{ Carbohydrate (\%) } & $\leq 65 \%$ & 912 & 64.1 & 13.3 & 49.2 & 22.8 & 14.8 & 0.921 \\
\hline & $>65 \%$ & 510 & 35.9 & 12.8 & 48.1 & 24.5 & 14.6 & \\
\hline \multirow{2}{*}{ Fat $(\%)$} & $\leq 30 \%$ & 1082 & 76.1 & 13.3 & 49.3 & 24.1 & 13.3 & 0.162 \\
\hline & $>30 \%$ & 340 & 23.9 & 12.3 & 47.4 & 21.3 & 18.9 & \\
\hline \multirow{2}{*}{ Saturated fat (\%) } & $<8 \%$ & 814 & 57.2 & 14.3 & 48.5 & 23.9 & 13.3 & 0.273 \\
\hline & $\geq 8 \%$ & 608 & 42.8 & 11.5 & 49.3 & 22.7 & 16.6 & \\
\hline \multirow{2}{*}{ Protein $(\%)$} & $<$ EAR & 223 & 15.7 & 12.8 & 52.8 & 20.3 & 14.0 & 0.615 \\
\hline & $\geq \mathrm{EAR}$ & 1199 & 84.3 & 13.1 & 48.0 & 24.0 & 14.8 & \\
\hline \multirow{2}{*}{ Fiber (\%) } & $<\mathrm{AI}$ & 1030 & 72.4 & 13.7 & 48.7 & 24.5 & 13.1 & 0.069 \\
\hline & $\geq \mathrm{AI}$ & 392 & 27.6 & 11.6 & 49.1 & 20.5 & 18.8 & \\
\hline \multirow{2}{*}{ Vitamin A (\%) } & $<$ EAR & 781 & 54.9 & 13.8 & 47.4 & 23.9 & 14.9 & 0.727 \\
\hline & $\geq \mathrm{EAR}$ & 641 & 45.1 & 12.2 & 50.7 & 22.7 & 14.4 & \\
\hline \multirow{2}{*}{ Vitamin C (\%) } & $<$ EAR & 966 & 67.9 & 14.9 & 47.9 & 23.1 & 14.1 & 0.040 * \\
\hline & $\geq \mathrm{EAR}$ & 456 & 32.1 & 9.1 & 50.9 & 24.0 & 16.0 & \\
\hline \multirow{2}{*}{ Sodium (\%) } & $<\mathrm{AI}$ & 144 & 10.1 & 12.9 & 51.4 & 22.7 & 13.1 & 0.927 \\
\hline & $\geq \mathrm{AI}$ & 1278 & 89.9 & 13.1 & 48.5 & 23.5 & 14.9 & \\
\hline \multirow{2}{*}{ Potassium (\%) } & $<\mathrm{AI}$ & 1116 & 78.5 & 13.7 & 48.1 & 24.3 & 13.9 & 0.158 \\
\hline & $\geq \mathrm{AI}$ & 306 & 21.5 & 10.7 & 51.7 & 20.0 & 17.6 & \\
\hline \multirow{2}{*}{ Calcium (\%) } & $<$ EAR & 1177 & 82.8 & 13.4 & 48.7 & 24.0 & 13.9 & 0.276 \\
\hline & $\geq \mathrm{EAR}$ & 245 & 17.2 & 11.6 & 49.6 & 20.4 & 18.4 & \\
\hline \multirow{2}{*}{ Phosphorus (\%) } & $<$ EAR & 667 & 46.9 & 15.2 & 49.3 & 21.7 & 13.7 & 0.127 \\
\hline & $\geq \mathrm{EAR}$ & 755 & 53.1 & 11.1 & 48.4 & 25.0 & 15.6 & \\
\hline \multirow{2}{*}{ Iron $(\%)$} & $<$ EAR & 548 & 38.5 & 15.7 & 46.0 & 24.0 & 14.3 & 0.161 \\
\hline & $\geq \mathrm{EAR}$ & 874 & 61.5 & 11.4 & 50.6 & 23.0 & 14.9 & \\
\hline \multirow{2}{*}{ VitaminB1 (\%) } & $<$ EAR & 145 & 10.2 & 18.4 & 51.8 & 22.7 & 7.1 & $0.035 *$ \\
\hline & $\geq \mathrm{EAR}$ & 1277 & 89.8 & 12.4 & 48.5 & 23.5 & 15.6 & \\
\hline \multirow{2}{*}{ Vitamin B2 (\%) } & $<$ EAR & 545 & 38.3 & 15.6 & 47.3 & 24.7 & 12.4 & 0.074 \\
\hline & $\geq \mathrm{EAR}$ & 877 & 61.7 & 11.5 & 49.8 & 22.5 & 16.2 & \\
\hline \multirow{2}{*}{ Niacin (\%) } & $<$ EAR & 464 & 32.6 & 15.6 & 48.8 & 23.8 & 11.8 & 0.109 \\
\hline & $\geq$ EAR & 958 & 67.4 & 11.8 & 48.8 & 23.2 & 16.2 & \\
\hline
\end{tabular}

Values are presented as the mean \pm standard error or percentage. AI, Adequate Intake; EAR, Estimated Average Requirements; ${ }^{*} P<0.05$. 
Table 3. Association between sleep duration and dietary nutrient intake.

\begin{tabular}{|c|c|c|c|c|c|c|}
\hline \multirow{2}{*}{$\begin{array}{c}\text { Total N } \\
\begin{array}{c}\text { Sleep Duration } \\
\text { (h/night) }\end{array}\end{array}$} & \multicolumn{6}{|c|}{ Total $n=1422$} \\
\hline & & $\begin{array}{c}<6 h \\
(n=157)\end{array}$ & $\begin{array}{l}6 \text { to }<8 h \\
(n=690)\end{array}$ & $\begin{array}{l}8 \text { to }<9 h \\
(n=362)\end{array}$ & $\begin{array}{c}\geq 9 h \\
(n=216)\end{array}$ & $p$ for Trends \\
\hline Nutrients (Unit) & & Mean & Mean & Mean & Mean & \\
\hline \multirow{3}{*}{ Carbohydrate (\%) } & model 1 & $61.1 \pm 0.9$ & $61.1 \pm 0.4$ & $61.5 \pm 0.7$ & $60.0 \pm 0.8$ & 0.446 \\
\hline & model 2 & $61.8 \pm 0.9$ & $61.5 \pm 0.4$ & $61.4 \pm 0.7$ & $59.8 \pm 0.8$ & 0.137 \\
\hline & model 3 & $61.1 \pm 1.1$ & $61.1 \pm 0.9$ & $61.0 \pm 0.9$ & $59.6 \pm 1.1$ & 0.221 \\
\hline \multirow{3}{*}{ Protein (\%) } & model 1 & $14.8 \pm 0.4$ & $14.4 \pm 0.2$ & $14.4 \pm 0.3$ & $14.3 \pm 0.3$ & 0.282 \\
\hline & model 2 & $14.6 \pm 0.4$ & $14.3 \pm 0.2$ & $14.5 \pm 0.3$ & $14.3 \pm 0.3$ & 0.752 \\
\hline & model 3 & $14.4 \pm 0.5$ & $14.1 \pm 0.4$ & $14.3 \pm 0.4$ & $14.0 \pm 0.5$ & 0.558 \\
\hline \multirow{3}{*}{ Fat (\%) } & model 1 & $24.1 \pm 0.8$ & $24.5 \pm 0.3$ & $24.1 \pm 0.5$ & $25.7 \pm 0.7$ & 0.185 \\
\hline & model 2 & $23.7 \pm 0.8$ & $24.2 \pm 0.3$ & $24.1 \pm 0.6$ & $25.8 \pm 0.7$ & 0.084 \\
\hline & model 3 & $24.4 \pm 1.0$ & $24.8 \pm 0.7$ & $24.8 \pm 0.8$ & $26.3 \pm 1.0$ & 0.109 \\
\hline \multirow{3}{*}{$\begin{array}{c}\text { Carbohydrate } \\
\text { (g/day) }\end{array}$} & model 1 & $305.9 \pm 13.2$ & $335.9 \pm 6.2$ & $328.8 \pm 7.5$ & $333.6 \pm 11.2$ & 0.152 \\
\hline & model 2 & $319.7 \pm 13.3$ & $337.2 \pm 5.7$ & $319.3 \pm 7.1$ & $328.0 \pm 11.6$ & 0.900 \\
\hline & model 3 & $322.6 \pm 7.3$ & $324.7 \pm 6.0$ & $316.2 \pm 7.6$ & $320.4 \pm 6.5$ & 0.553 \\
\hline \multirow{3}{*}{ Protein (g/day) } & model 1 & $75.9 \pm 4.9$ & $82.1 \pm 2.2$ & $78.6 \pm 2.6$ & $79.6 \pm 3.1$ & 0.660 \\
\hline & model 2 & $77.1 \pm 4.8$ & $81.2 \pm 2.0$ & $76.3 \pm 2.5$ & $78.9 \pm 3.3$ & 0.976 \\
\hline & model 3 & $79.2 \pm 3.3$ & $78.4 \pm 2.4$ & $76.4 \pm 2.8$ & $76.9 \pm 2.9$ & 0.367 \\
\hline \multirow{3}{*}{ Fat (g/day) } & model 1 & $57.7 \pm 4.6$ & $62.9 \pm 1.8$ & $60.1 \pm 2.5$ & $64.3 \pm 3.2$ & 0.328 \\
\hline & model 2 & $58.2 \pm 4.7$ & 62.1 & $58.5 \pm 2.4$ & $63.8 \pm 3.4$ & 0.496 \\
\hline & model 3 & $62.5 \pm$ & 62.4 & $61.0 \pm 2.1$ & $64.7 \pm 2.3$ & 0.547 \\
\hline \multirow{3}{*}{$\begin{array}{l}\text { Saturated fat } \\
\text { (g/day) }\end{array}$} & model 1 & $18.0 \pm 1.4$ & $20.0 \pm 0.6$ & $19.4 \pm 0.8$ & $20.7 \pm 1.2$ & 0.164 \\
\hline & model 2 & $18.5 \pm 1.4$ & $19.8 \pm 0.5$ & $18.8 \pm 0.8$ & $20.5 \pm 1.2$ & 0.398 \\
\hline & model 3 & $19.0 \pm 0.9$ & $19.1 \pm 0.7$ & $18.8 \pm 0.8$ & $20.0 \pm 1.1$ & 0.463 \\
\hline \multirow{3}{*}{ Cholesterol (mg/d) } & model 1 & $273.5 \pm 21.0$ & $326.7 \pm 11.3$ & $299.6 \pm 14.0$ & $324.3 \pm 24.1$ & 0.184 \\
\hline & model 2 & $271.8 \pm 22.5$ & $322.3 \pm 10.7$ & $294.2 \pm 14.0$ & $323.8 \pm 24.4$ & 0.214 \\
\hline & model 3 & $263.8 \pm 19.1$ & $293.0 \pm 16.6$ & $275.2 \pm 17.7$ & $297.7 \pm 24.6$ & 0.307 \\
\hline \multirow{3}{*}{ Fiber (g/day) } & model 1 & $16.7 \pm 0.9$ & $18.5 \pm 0.4$ & $18.4 \pm 0.6$ & $20.6 \pm 1.2$ & $0.008 *$ \\
\hline & model 2 & $17.1 \pm 0.9$ & $18.5 \pm 0.4$ & $18.0 \pm 0.6$ & $20.4 \pm 1.2$ & $0.039 *$ \\
\hline & model 3 & $16.5 \pm 0.9$ & $17.0 \pm 0.7$ & $17.0 \pm 0.8$ & $19.3 \pm 1.0$ & $0.022 *$ \\
\hline \multirow{3}{*}{ Vitamin A (mcg/d) } & model 1 & $580.2 \pm 62.0$ & $670.7 \pm 33.4$ & $680.2 \pm 54.5$ & $730.4 \pm 134.4$ & 0.307 \\
\hline & model 2 & $630.3 \pm 70.9$ & $682.4 \pm 35.4$ & $656.7 \pm 55.0$ & $712.3 \pm 129.0$ & 0.584 \\
\hline & model 3 & $579.5 \pm 71.6$ & $593.9 \pm 47.1$ & $576.2 \pm 73.1$ & $628.2 \pm 117.0$ & 0.748 \\
\hline \multirow{3}{*}{ Vitamin C (mg/d) } & model 1 & $59.7 \pm 6.6$ & $77.4 \pm 4.6$ & $74.2 \pm 5.1$ & $77.1 \pm 6.1$ & 0.078 \\
\hline & model 2 & $63.9 \pm 6.7$ & $78.5 \pm 4.3$ & $72.4 \pm 5.3$ & $75.6 \pm 6.0$ & 0.311 \\
\hline & model 3 & $61.0 \pm 9.6$ & $73.8 \pm 11.2$ & $70.1 \pm 10.7$ & $73.4 \pm 10.3$ & 0.248 \\
\hline \multirow{3}{*}{ Sodium (mg/d) } & model 1 & $3704.1 \pm 245.9$ & $3701.6 \pm 106.7$ & $3431.3 \pm 109.2$ & $3429.2 \pm 148.5$ & 0.170 \\
\hline & model 2 & $3734.7 \pm 238.4$ & $3658.2 \pm 98.0$ & $3338.5 \pm 103.6$ & $3403.7 \pm 156.9$ & 0.112 \\
\hline & model 3 & $3890.9 \pm 172.4$ & $3638.4 \pm 126.1$ & $3436.6 \pm 139.9$ & $3433.2 \pm 161.1$ & $0.005 *$ \\
\hline \multirow{3}{*}{ Potassium (mg/d) } & model 1 & $2401.2 \pm 120.1$ & $2715.4 \pm 57.8$ & $2597.2 \pm 77.8$ & $2800.8 \pm 128.7$ & 0.039 \\
\hline & model 2 & $2487.0 \pm 124.2$ & $2716.5 \pm 53.1$ & $2527.6 \pm 75.0$ & $2764.4 \pm 132.0$ & 0.243 \\
\hline & model 3 & $2446.4 \pm 100.1$ & $2532.1 \pm 75.8$ & $2410.7 \pm 80.8$ & $2607.5 \pm 107.4$ & 0.413 \\
\hline \multirow{3}{*}{ Calcium (mg/d) } & model 1 & $434.1 \pm 24.4$ & $483.5 \pm 10.8$ & $489.1 \pm 18.0$ & $555.3 \pm 47.4$ & 0.015 \\
\hline & model 2 & $454.5 \pm 25.9$ & $486.5 \pm 10.6$ & $476.7 \pm 17.7$ & $547.4 \pm 48.7$ & 0.127 \\
\hline & model 3 & $437.8 \pm 27.5$ & $448.7 \pm 22.5$ & $451.8 \pm 25.4$ & $521.3 \pm 55.1$ & 0.129 \\
\hline \multirow{3}{*}{$\begin{array}{l}\text { Phosphorus } \\
(\mathrm{mg} / \mathrm{d})\end{array}$} & model 1 & $1015.4 \pm 49.0$ & $1136.5 \pm 24.1$ & $1114.8 \pm 32.7$ & $1154.8 \pm 42.1$ & 0.045 \\
\hline & model 2 & $1052.8 \pm 51.5$ & $1136.1 \pm 22.1$ & $1083.3 \pm 31.7$ & $1138.7 \pm 44.3$ & 0.357 \\
\hline & model 3 & $1033.0 \pm 28.6$ & $1052.0 \pm 23.4$ & $1031.7 \pm 26.4$ & $1068.4 \pm 32.6$ & 0.448 \\
\hline \multirow{3}{*}{ Iron $(\mathrm{mg} / \mathrm{d})$} & model 1 & $14.9 \pm 1.1$ & $16.6 \pm 0.6$ & $18.4 \pm 2.7$ & $15.4 \pm 0.7$ & 0.506 \\
\hline & model 2 & $15.5 \pm 1.4$ & $16.6 \pm 0.6$ & $17.9 \pm 2.5$ & $15.2 \pm 0.7$ & 0.947 \\
\hline & model 3 & $15.8 \pm 1.9$ & $16.0 \pm 1.1$ & $17.8 \pm 2.6$ & $14.9 \pm 1.2$ & 0.822 \\
\hline
\end{tabular}


Table 3. Cont

\begin{tabular}{|c|c|c|c|c|c|c|}
\hline \multirow{2}{*}{$\begin{array}{c}\text { Total N } \\
\begin{array}{c}\text { Sleep Duration } \\
\text { (h/night) }\end{array}\end{array}$} & \multicolumn{6}{|c|}{ Total $n=1422$} \\
\hline & & $\begin{array}{c}<6 h \\
(n=157)\end{array}$ & $\begin{array}{l}6 \text { to }<8 h \\
(n=690)\end{array}$ & $\begin{array}{l}8 \text { to }<9 h \\
(n=362)\end{array}$ & $\begin{array}{c}\geq 9 h \\
(n=216)\end{array}$ & $p$ for Trends \\
\hline Nutrients (Unit) & & Mean & Mean & Mean & Mean & \\
\hline \multirow{3}{*}{ Vitamin B1(mg/d) } & model 1 & $1.96 \pm 0.13$ & $2.14 \pm 0.05$ & $2.00 \pm 0.06$ & $2.08 \pm 0.08$ & 0.654 \\
\hline & model 2 & $1.98 \pm 0.13$ & $2.11 \pm 0.05$ & $1.95 \pm 0.06$ & $2.06 \pm 0.08$ & 0.882 \\
\hline & model 3 & $2.03 \pm 0.12$ & $2.06 \pm 0.11$ & $1.96 \pm 0.10$ & $2.03 \pm 0.10$ & 0.662 \\
\hline \multirow{3}{*}{ Vitamin B6 (mg/d) } & model 1 & $1.32 \pm 0.07$ & $1.50 \pm 0.03$ & $1.46 \pm 0.06$ & $1.51 \pm 0.06$ & 0.057 \\
\hline & model 2 & $1.35 \pm 0.08$ & $1.50 \pm 0.03$ & $1.42 \pm 0.06$ & $1.49 \pm 0.06$ & 0.306 \\
\hline & model 3 & $1.36 \pm 0.09$ & $1.42 \pm 0.08$ & $1.38 \pm 0.08$ & $1.42 \pm 0.08$ & 0.501 \\
\hline \multirow{3}{*}{ Niacin $(\mathrm{mcg} / \mathrm{d})$} & model 1 & $15.99 \pm 1.19$ & $16.75 \pm 0.47$ & $16.28 \pm 0.73$ & $16.40 \pm 0.64$ & 0.853 \\
\hline & model 2 & $16.13 \pm 1.17$ & $16.55 \pm 0.45$ & $15.85 \pm 0.71$ & $16.29 \pm 0.67$ & 0.960 \\
\hline & model 3 & $16.60 \pm 0.91$ & $15.97 \pm 0.63$ & $15.84 \pm 0.62$ & $15.85 \pm 0.72$ & 0.347 \\
\hline
\end{tabular}

Values are presented as the mean \pm standard error or percentage, ${ }^{*} P<0.01$.

\section{Discussions}

The key finding of this national population-based study was the association between nutrients and sleep duration among adolescents. Particularly, shorter sleep duration was associated with decreased intake of fiber and increased intake of sodium. In addition, intake of vitamin B1 and C was associated with sleep duration.

\subsection{Sleep Duration Associated with Obesity}

In this study, we found an inverse association between sleep duration and obese status in South Korean adolescents, which is consistent with previous epidemiological studies performed in children and adolescents [23,24].

There are two most commonly reported associations between sleep duration and obesity. The first is an inverse linear correspondence where shorter sleep durations are associated with increased risk of obesity. This pattern suggests that more sleep is better for preventing obesity. In addition, a meta-analysis by Chen et al. has reported a linear dose-response association in children aged $<10$ years; however, studies in adolescents have inconsistent results [25]. In an 8-year prospective cohort study, shorter sleep duration was more strongly associated with a higher BMI in middle childhood than in adolescence [26].

The other pattern is a U-shaped association in which sleep durations that are longer or shorter than about $7-8 \mathrm{~h} /$ night were associated with increased risk of obesity $[27,28]$.

The difference between these two sleep patterns may be related to whether the sleep needs are met according with age. Most adults need about 7-8 h of sleep per day, but infants and children need more. Physiologically, the younger the age, the longer the sleep need; children have a long sleep duration. However, adults have less sleep needs than children, but they often sleep less than they need to. Instead, long sleepers are often reported as mentally ill. Therefore, it is possible that insufficient sleep or excess sleep may explain the U-shaped association between sleep and obesity in older age. Future studies should investigate these differences by comparing different age groups.

\subsection{Sleep Duration and Dietary Nutrients}

Our findings indicate that sleep duration may be associated with intake of fiber and vitamins, but not with fat, protein and carbohydrate It is still unclear whether sleep duration affects dietary intake or dietary intake affects sleep duration and quality. Some studies on the association between sleep duration and dietary nutrients have yielded different findings in adults. Changed dietary pattern after sleep deprivation is commonly associated with macronutrients such as fat or carbohydrates. In one study, the diets of adolescents aged 14-16 years after several nights of sleep restriction were characterized by higher glycemic index and glycemic load and a trend toward increased consumption 
of calories and carbohydrates [29]. In another study, older adolescents sleeping $<8 \mathrm{~h}$ consumed a higher proportion of calories from fat and a lower proportion of calories from carbohydrates [30]. Moreover, pubertal insulin resistance is physiological, occurring with pubertal progression and resolving by the end of puberty, and is associated with decreased peripheral insulin sensitivity and increased insulin secretion [31,32]. Hyperinsulinemia is a marker of obesity and reduced SWS has been shown to influence insulin insensitivity in young adults [33]. Therefore, insufficient sleep may have a greater effect on obesity during adolescence than during childhood.

Nutrients affect sleep architecture and pattern. A recent study reported that greater fiber intake predicted less sleep stage 1 and more SWS, and low fiber intake was associated with arousals in polysomnography of adults. [34]. Moreover, carbohydrates and proteins can influence the levels of neurotransmitters in the intrinsic sleep processes and affect sleep patterns. For example, short-term low proportion of carbohydrate intake was found to increase the percentage of SWS and reduce the percentage of REM sleep among healthy sleepers [35].

Vitamin $C$ is an important antioxidant found in citrus fruits and vegetables. Low intake of vitamin $C$ is associated with non-restorative sleep [36]. Vitamin B is involved in many metabolic functions, including sleep and circadian factors. Vitamin B has been shown to reduce daytime sleepiness and improve sleep pattern. A large study found that normal sleepers ingested significantly higher levels of vitamin B1 and vitamin B12 than those with insomnia [37]. Additionally, vitamin B1 deficiency has been associated with short sleep duration in adults, and adults who sleep $<6 \mathrm{~h}$ have been shown to have a significantly lower intake of potassium, fiber, and calcium [38].

There have been studies supporting the association between fruit and vegetable intake and sleep dduration, with one indicating that adolescents who sleep $>8 \mathrm{~h}$ consume more fruit and vegetables [39], and another showing that $<7 \mathrm{~h}$ self-reported sleep is associated with reduced vegetable and fruit consumption compared with $>8$ h sleep duration [40]. Fruits and vegetables are rich in vitamins and dietary fiber. These studies support our findings and suggest that food choices may play a role in sleep duration in adolescents.

Our study found an adverse association between sodium intake and sleep duration in adolescents. Sodium intake is associated with blood pressure, and elevated blood pressure is associated with short sleep duration and poor efficacy in adolescents and adults [41,42]. A national study has reported that salt is associated with sleep maintenance difficulties in adults (odds ratio $=1.19$ ) [43]. The biological mechanisms underlying the association between short sleep duration and hypertension are unclear. Our finding regarding short sleep duration in a group with high intake of sodium may illustrate a possible link between insufficient sleep and hypertension.

Diet could be useful in the management of sleep disturbances. It may be a self-feeding mechanism where dietary intake affects sleep pattern, which then affects food choices and leads to alterations in dietary consumption patterns.

\subsection{Study Limitations and Strengths}

This study has several limitations. First, this study was based on KNHANES data and the associations do not necessarily imply causation. Our results suggest only exploratory evidence which is obtained without controlling false positive rates statistically. Therefore, future confirmatory studies are needed to find solid evidence of relationships. Second, like most previous studies, this study depended on self-reported sleep duration and there is no distinction between weekends and weekdays. Studies on the correlation between self-reported sleep duration and objectively measured sleep duration have reported moderate correlation [44,45]. Finally, the dietary data were collected using a 24-h dietary recall, limiting the ability to measure usual intake. However, under-reporting is less likely in 24-h dietary recalls than in self-reporting surveys in which participants are asked to record their own food intake [46]. In addition, at a population level, it can provide rich details about the mean dietary intake for a given day [47] 
In conclusion, sleep duration can be associated with intake of some nutrients, which may also be associated with obesity in adolescents. Therefore, it is possible to prevent obesity and its complications by controlling the sleep duration and intake of nutrients of adolescents.

Author Contributions: Conceptualization, W.H.S.; methodology, S.-J.C.; formal analysis, S.-J.C.; investigation, J.H.L.; data curation, W.H.S., S.-J.C.; writing-original draft preparation, J.H.L.; writing-review and editing, W.H.S., S.-J.C.; visualization, J.H.L., S.-J.C. All authors have read and agreed to the published version of the manuscript.

Funding: This research received no external funding.

Acknowledgments: This research was supported by Korea University.

Conflicts of Interest: The authors declare no conflict of interest.

\section{References}

1. Colrain, I.M.; Baker, F.C. Changes in Sleep as a Function of Adolescent Development. Neuropsychol. Rev. 2011, 1, 5-21. [CrossRef] [PubMed]

2. Vernon, L.; Barber, B.L.; Modecki, K.L. Adolescent Problematic Social Networking and School Experiences: The Mediating Effects of Sleep Disruptions and Sleep Quality. Cyberpsychol. Behav. Soc. Netw. 2015, 18, 386-392. [CrossRef] [PubMed]

3. Short, M.A.; Weber, N.; Reynolds, C.; Coussens, S.; Carskadon, M.A. Estimating Adolescent Sleep Need Using Dose-Response Modeling. Sleep 2018, 41, zsy011. [CrossRef] [PubMed]

4. Fuligni, A.J.; Bai, S.; Krull, J.L.; Gonzales, N.A. Individual Differences in Optimum Sleep for Daily Mood During Adolescence. J. Clin. Child Adolesc. Psychol. 2019, 48, 469-479. [CrossRef] [PubMed]

5. Seo, S.H.; Shim, Y.S. Association of Sleep Duration with Obesity and Cardiometabolic Risk Factors in Children and Adolescents: A Population-Based Study. Sci. Rep. 2019, 9, 1-10. [CrossRef] [PubMed]

6. Wang, Y.; Lobstein, T. Worldwide Trends in Childhood Overweight and Obesity. Int. J. Pediatr. Obes. 2006, 1, 11-25. [CrossRef]

7. Sparano, S.; Lauria, F.; Ahrens, W.; Fraterman, A.; Thumann, B.; Iacoviello, L.; Marild, S.; Michels, N.; Molnar, D.; Moreno, L.A.; et al. Sleep Duration and Blood Pressure in Children: Analysis of the Pan-European IDEFICS Cohort. J. Clin. Hypertens. 2019, 21, 572-578. [CrossRef]

8. Hanlon, E.C.; Dumin, M.; Pannain, S. Sleep and Obesity in Children and Adolescents. In Global Perspectives on Childhood Obesity, 2nd ed.; Bagchi, D., Ed.; Elsevier: London, UK, 2019; pp. 147-178.

9. 2018 Global Nutrition Report: Shining a Light to Spur Action on Nutrition. 2018. Available online: https://www.who.int/nutrition/globalnutritionreport/2018_Global_Nutrition_Report.pdf (accessed on 3 September 2020).

10. Knutson, K.L. Sleep Duration and Cardiometabolic Risk: A Review of the Epidemiologic Evidence. Best. Pract. Res. Clin. Endocrinol. Metab. 2010, 24, 731-743. [CrossRef]

11. Chaput, J.-P. Short Sleep Duration Promoting Overconsumption of Food: A Reward-Driven Eating Behavior? Sleep 2010, 33, 1135-1136. [CrossRef]

12. Fu, J.; Wang, Y.; Li, G.; Han, L.; Li, Y.; Li, L.; Feng, D.; Wu, Y.; Xiao, X.; Li, M.; et al. Childhood Sleep Duration Modifies the Polygenic Risk for Obesity in Youth Through Leptin Pathway: The Beijing Child and Adolescent Metabolic Syndrome Cohort Study. Int. J. Obes. 2019, 43, 1556-1567. [CrossRef]

13. Matthews, K.A.; Dahl, R.E.; Owens, J.F.; Lee, L.; Hall, M. Sleep Duration and Insulin Resistance in Healthy Black and White Adolescents. Sleep 2012, 35, 1353-1358. [CrossRef]

14. Kim, W.; Lee, J.; Ha, J.; Jo, K.; Lim, D.-J.; Lee, J.-M.; Chang, S.-A.; Kang, M.-I.; Kim, M.-H. Association Between Sleep Duration and Subclinical Thyroid Dysfunction Based on Nationally Representative Data. Maedica 2019, 8, 2010.

15. Spiegel, K.; Leproult, R.; L’Hermite-Balériaux, M.; Copinschi, G.; Penev, P.D.; Van Cauter, E. Leptin Levels are Dependent on Sleep Duration: Relationships with Sympathovagal Balance, Carbohydrate Regulation, Cortisol, and Thyrotropin. J. Clin. Endocrinol. Metab. 2004, 89, 5762-5771. [CrossRef]

16. Katagiri, R.; Asakura, K.; Kobayashi, S.; Suga, H.; Sasaki, S. Low Intake of Vegetables, High Intake of Confectionary, and Unhealthy Eating Habits are Associated with Poor Sleep Quality among Middle-Aged Female Japanese Workers. J. Occup. Health 2014, 14, 359-368. [CrossRef] 
17. Jaussent, I.; Dauvilliers, Y.; Ancelin, M.-L.; Dartigues, J.-F.; Tavernier, B.; Touchon, J.; Ritchie, K.; Besset, A. Insomnia Symptoms in Older Adults: Associated Factors and Gender Differences. Am. J. Geriatr. Psychiatry 2011, 19, 88-97. [CrossRef] [PubMed]

18. Kweon, S.; Kim, Y.; Jang, M.j.; Kim, Y.; Kim, K.; Choi, S.; Chaemin, C.; Khang, Y.-H.; Oh, K. Data Resource Profile: The Korea National Health and Nutrition Examination Survey (KNHANES). Int. J. Epidemiol. 2014, 43, 69-77. [CrossRef]

19. Barlow, S.E. Expert Committee Recommendations Regarding the Prevention, Assessment, and Treatment of Child and Adolescent Overweight and Obesity: Summary Report. Pediatrics 2007, 120, S164-S192. [CrossRef]

20. Rural Resources Development Institute. Food Composition Table; 7 th revision; National Rural Resources Development Institute, Rural Development Administration: Suwon, Korea, 2006.

21. National Academy of Agricultural Sciences. Food Composition Table; 8 th revision; National Academy of Agricultural Sciences: Suwon, Korea, 2011; pp. 1-636.

22. MoHa, W. Dietary Reference Intakes for Koreans 2015; Ministry of Health and Welfare: Sejong, Korea, 2015.

23. Tambalis, K.D.; Panagiotakos, D.B.; Psarra, G.; Sidossis, L.S. Insufficient Sleep Duration is Associated with Dietary Habits, Screen Time, and Obesity in Children. J. Clin. Sleep Med. 2018, 14, 1689-1696. [CrossRef]

24. Cao, M.; Zhu, Y.; He, B.; Yang, W.; Chen, Y.; Ma, J.; Jing, J. Association Between Sleep Duration and Obesity is Age-and Gender-Dependent in Chinese Urban Children Aged 6-18 Years: A Cross-Sectional Study. BMC Public Health. 2015, 15, 1029. [CrossRef]

25. Chen, X.; Beydoun, M.A.; Wang, Y. Is Sleep Duration Associated with Childhood Obesity? A Systematic Review and Meta-Analysis. Obesity 2008, 16, 265-274. [CrossRef]

26. Storfer-Isser, A.; Patel, S.R.; Babineau, D.C.; Redline, S. Relation Between Sleep Duration and BMI Varies by Age and Sex in Youth Age 8-19. Pediatr. Obes. 2012, 7, 53-64. [CrossRef]

27. Smiley, A.; King, D.; Bidulescu, A. The Association between Sleep Duration and Metabolic Syndrome: The NHANES 2013/2014. Nutrients 2019, 11, 2582. [CrossRef]

28. Jean-Louis, G.; Williams, N.J.; Sarpong, D.; Pandey, A.; Youngstedt, S.; Zizi, F.; Ogedegbe, G. Associations between Inadequate Sleep and Obesity in the US Adult Population: Analysis of the National Health Interview Survey (1977-2009). BMC Public Health 2014, 14, 290. [CrossRef]

29. Beebe, D.W.; Simon, S.; Summer, S.; Hemmer, S.; Strotman, D.; Dolan, L.M. Dietary Intake Following Experimentally Restricted Sleep in Adolescents. Sleep 2013, 36, 827-834. [CrossRef]

30. Weiss, A.; Xu, F.; Storfer-Isser, A.; Thomas, A.; Ievers-Landis, C.E.; Redline, S. The Association of Sleep Duration with Adolescents' Fat and Carbohydrate Consumption. Sleep 2010, 33, 1201-1209. [CrossRef]

31. Androutsos, O.; Moschonis, G.; Mavrogianni, C.; Roma-Giannikou, E.; Chrousos, G.; Kanaka-Gantenbein, C.; Manios, Y. Identification of Lifestyle Patterns, including Sleep Deprivation, Associated with Insulin Resistance in Children: The Healthy Growth Study. Eur. J. Clin. Nutr. 2014, 68, 344-349. [CrossRef]

32. Hannon, T.S.; Janosky, J.; Arslanian, S.A. Longitudinal Study of Physiologic Insulin Resistance and Metabolic Changes of Puberty. Pediatr. Res. 2006, 60, 759-763. [CrossRef]

33. Tasali, E.; Leproult, R.; Ehrmann, D.A.; Van Cauter, E. Slow-Wave Sleep and the Risk of Type 2 Diabetes in Humans. Proc. Natl. Acad. Sci. USA. 2008, 105, 1044-1049. [CrossRef]

34. St-Onge, M.-P.; Roberts, A.; Shechter, A.; Choudhury, A.R. Fiber and Saturated Fat are Associated with Sleep Arousals and Slow Wave Sleep. J. Clin. Sleep Med. 2016, 12, 19-24. [CrossRef]

35. Afaghi, A.; O'Connor, H.; Chow, C.M. Acute Effects of the Very Low Carbohydrate Diet on Sleep Indices. Nutr. Neurosci. 2008, 11, 146-154. [CrossRef]

36. Khan, M.K.; Faught, E.L.; Chu, Y.L.; Ekwaru, J.P.; Storey, K.E.; Veugelers, P.J. Is it Nutrients, Food Items, Diet Quality or Eating Behaviours That are Responsible for the Association of Children's Diet with Sleep? J. Sleep Res. 2017, 26, 468-476. [CrossRef]

37. Zadeh, S.S.; Begum, K. Comparison of Nutrient Intake by Sleep Status in Selected Adults in Mysore, India. Nutr. Res. Pract. 2011, 5, 230-235. [CrossRef]

38. Grandner, M.A.; Jackson, N.; Gerstner, J.R.; Knutson, K.L. Dietary Nutrients Associated with Short and Long Sleep Duration. Data from a Nationally Representative Sample. Appetite 2013, 64, 71-80. [CrossRef]

39. Garaulet, M.; Ortega, F.; Ruiz, J.; Rey-Lopez, J.; Beghin, L.; Manios, Y.; Cuenca-Garcia, M.; Plada, M.; Diethelm, K.; Kafatos, A.; et al. Short Sleep Duration is Associated with Increased Obesity Markers in European Adolescents: Effect of Physical Activity and Dietary Habits. The HELENA Study. Int. J. Obes. 2011, 35, 1308. [CrossRef] 
40. Kruger, A.K.; Reither, E.N.; Peppard, P.E.; Krueger, P.M.; Hale, L. Do Sleep-Deprived adolescents Make Less-Healthy Food Choices? Br. J. Nutr. 2014, 111, 1898-1904. [CrossRef] [PubMed]

41. Gottlieb, D.J.; Redline, S.; Nieto, F.J.; Baldwin, C.M.; Newman, A.B.; Resnick, H.E.; Punjabi, N.M. Association of Usual Sleep Duration with Hypertension: The Sleep Heart Health Study. Sleep 2006, 29, 1009-1014. [CrossRef]

42. Javaheri, S.; Storfer-Isser, A.; Rosen, C.L.; Redline, S. Sleep Quality and Elevated Blood Pressure in Adolescents. Circulation 2008, 118, 1034-1040. [CrossRef]

43. Grandner, M.A.; Jackson, N.; Gerstner, J.R.; Knutson, K.L. Sleep Symptoms Associated with Intake of Specific Dietary Nutrients. J. Sleep Res. 2014, 23, 22-34. [CrossRef]

44. Lauderdale, D.S.; Knutson, K.L.; Yan, L.L.; Liu, K.; Rathouz, P.J. Self-Reported and Measured Sleep Duration: How Similar are They? Epidemiology 2008, 19, 838-845. [CrossRef]

45. Cespedes, E.M.; Hu, F.B.; Redline, S.; Rosner, B.; Alcantara, C.; Cai, J.; Hall, M.H.; Loredo, J.S.; Mossavar-Rahmani, Y.; Ramos, A.R.; et al. Comparison of Self-Reported Sleep Duration with Actigraphy: Results from the Hispanic Community Health Study/Study of Latinos Sueño Ancillary Study. Epidemiol. Rev. 2016, 183, 561-573. [CrossRef] [PubMed]

46. Johnson, R.K.; Driscoll, P.; Goran, M.I. Comparison of Multiple-Pass 24-Hour Recall Estimates of Energy Intake with Total Energy Expenditure Determined by the Doubly Labeled Water Method in Young Children. J. Am. Diet. Assoc. 1996, 96, 1140-1144. [CrossRef]

47. Ahluwalia, N.; Dwyer, J.; Terry, A.; Moshfegh, A.; Johnson, C. Update on NHANES Dietary Data: Focus on Collection, Release, Analytical Considerations, and Uses to Inform Public Policy. Adv. Nutr. 2016, 7, 121-134. [CrossRef]

Publisher's Note: MDPI stays neutral with regard to jurisdictional claims in published maps and institutional affiliations. 